\title{
Proteomic Biomarkers of Intra-amniotic Inflammation: Relationship with Funisitis and Early-onset Sepsis in the Premature Neonate
}

\author{
CATALIN S. BUHIMSCHI, IRINA A. BUHIMSCHI, SONYA ABDEL-RAZEQ, VICTOR A. ROSENBERG, STEPHEN F. THUNG, \\ GUOMAO ZHAO, ERICA WANG, AND VINEET BHANDARI \\ Department of Obstetrics, Gynecology and Reproductive Sciences [C.S.B., I.A.B., S.A.-R., V.A.R., S.F.T., G.Z., E.W.], and Department of
Pediatrics [V.B.], Division of Perinatal Medicine, Yale University School of Medicine, New Haven, CT 06520
}

\begin{abstract}
Our goal was to determine the relationship between 4 amniotic fluid (AF) proteomic biomarkers (human neutrophil defensins 2 and 1, calgranulins $\mathrm{C}$ and A) characteristic of intra-amniotic inflammation, and funisitis and early-onset sepsis in premature neonates. The mass restricted (MR) score was generated from AF obtained from women in preterm labor $(n=123)$. The MR score ranged from $0-4$ (none to all biomarkers present). Funisitis was graded histologically and interpreted in relation to the MR scores. Neonates $(n=97)$ were evaluated for early-onset sepsis. There was significant correlation between the severity of AF inflammation and the presence $(53 / 123)$ and grades of funisitis $(p<0.001)$. Funisitis occurred independently of the amniocentesis-to-delivery interval or status of the membranes and was best predicted by an MR score 3-4 and an earlier gestational age (GA) at delivery. Neonates born to women with an MR score 3-4 had an increased incidence of suspected/confirmed sepsis, even after adjusting for GA at birth. Calgranulin $\mathrm{C}$ had the highest association with clinically significant funisitis, while calgranulin A had the strongest association with early-onset sepsis. To conclude, AF proteomic analysis shows that women with MR scores 3-4 are more likely to have histologic funisitis, and deliver neonates with early-onset sepsis. (Pediatr Res 61: 318-324, 2007)
\end{abstract}

D espite extensive research and a variety of interventions, the rate of preterm birth (PTB) has actually increased over the past $20 \mathrm{y}(1)$. More than 500,000 preterm infants were born in the United States in 2004 (1). Interventions to prevent PTB and its consequences for the fetus should predominantly be targeted at the prevention, early recognition and treatment of several risk factors including intra-amniotic inflammation/ infection (2).

Robust scientific evidence has confirmed that when intraamniotic inflammation secondary to, or independent of infection is superimposed upon prematurity, the consequences for the neonate can prove devastating $(3,4)$. This association can be explained by an inflammatory cytokine cascade that seems to be, at least partially, of fetal origin $(5,6)$. Indeed, fetal umbilical

Received June 30, 2006; accepted October 6, 2006

Correspondence: Vineet Bhandari, M.D., Yale University School of Medicine, Division of Perinatal Medicine, Department of Pediatrics, 333 Cedar Street, LCI 401B, New Haven, CT 06520; e-mail: vineet.bhandari@yale.edu

Supported by NICHD RO3 HD 50249-01 and K12 HD 047018-01 WRHR (C.S.B.); NICHD RO1 HD 047321 (I.A.B.); and NHLBI K08 HL 074195 (V.B.).

DOI: 10.1203/01.pdr.0000252439.48564.37 vein inflammatory cytokine levels, but not maternal serum values, correlate with the presence and severity of the placental histologic inflammation and umbilical cord vasculitis (7).

Funisitis is characterized by perivascular infiltrates of inflammatory cells and is considered one of the strongest hallmarks of microbial invasion of the amniotic cavity and fetal inflammatory syndrome $(8,9)$. While there is some debate with regard to the origin of the amniotic fluid (AF) neutrophilic response to infection (10), there is consensus that the fetal inflammatory neutrophilic response originates initially from the umbilical vein and later from the arteries leading to umbilical cord vasculitis and funisitis (11). Funisitis is a well-recognized risk factor for early-onset sepsis in the premature neonate $(12,13)$.

We have previously shown that proteomic mapping of the $\mathrm{AF}$ reveals a profile, designated as the Mass Restricted (MR) score, that is highly characteristic of intra-amniotic inflammation (14). The presence of 4 protein biomarkers (neutrophil defensins 2 and 1, calgranulins $\mathrm{C}$ and $\mathrm{A}$ ) were highly predictive of PTB, whether inflammation was secondary to infection or bleeding (15). We further found that the interpretation of the MR score is not binary (disease present or disease absent), but rather a gradient progressing from the absence of disease, to minimal, and then severe inflammation. Therefore, grading the severity of umbilical cord inflammation with increasing numerical values of the MR score was based on the number (from 1 to 4) of protein biomarkers being identified in the AF. Thus far, the relationship between the MR score or the individual 4 proteomic biomarkers, and histologic funisitis has not been addressed. In the current study, we sought to determine the relationship between presence in the $\mathrm{AF}$ of 4 proteomic biomarkers characteristic of intra-amniotic inflammation, and the likelihood of histologic funisitis and early-onset sepsis in premature neonates.

\section{METHODS}

Patient population and study design. We conducted a prospective study that included 123 women recruited upon admission to the Labor and Birth unit or the antepartum units at Yale New Haven Hospital. For all participants, following admission with symptoms of preterm labor (PTL), preterm prema-

Abbreviations: AF, amniotic fluid; LDH, lactate dehydrogenase; MR score, mass restricted score; PPROM, preterm premature rupture of membranes; PTB, pretem birth; PTL, preterm labor; WBC, white blood cells 
ture rupture of membranes (PPROM), advanced cervical dilatation $(\geq 3 \mathrm{~cm}$ ) and/or uterine contractions refractory to tocolysis, AF was retrieved by ultrasound-guided amniocentesis. Subjects were enrolled (May 2004-May 2006) based on the availability of one of the investigators (CSB), and all enrolled women were included in the final analysis.

The Human Investigation Committee of Yale University approved the study and written informed consent was obtained from all participants. Eligible subjects had: a singleton fetus, gestational age $(\mathrm{GA})<37$ wk with a clinically indicated amniocentesis to rule-out intra-amniotic infection. Exclusion criteria included: anhydramnios, human immunodeficiency virus or hepatitis infections, and the presence of fetal heart rate abnormalities at enrollment (bradycardia, or prolonged variable decelerations). GA was established based on an ultrasonographic examination before $20 \mathrm{wk}$ in all instances. PTL was defined as the presence of regular uterine contractions and documented cervical effacement and/or dilatation in patients $<37$ wk GA (16). The diagnosis of PPROM was confirmed by visualization of AF pooling through the cervical os, nitrazine, ferning, or amniocentesis-dye positive tests. Management of the patients was left to the clinical team. In the absence of clinical laboratory results suggestive of infection, or signs/symptoms of clinical chorioamnionitis (fever $>100.4^{\circ} \mathrm{F}$, abdominal tenderness, fetal tachycardia), and/or abnormalities of fetal heart rate (variable or late decelerations), and/or abruption, PPROM was managed expectantly. In PPROM patients, digital exams were not permitted. Patients received corticosteroids for lung maturity if $<32$ wk GA and antibiotic therapy (latency phase: ampicillin/ erythromycin or clindamycin; group B Streptococcus prophylaxis: penicillin, cefazolin, clindamycin, erythromycin or vancomycin, based on culture results and sensitivity). Eighty percent of women had their amniocentesis performed before initiation of antibiotic or steroid therapy. Women were monitored by cardiotocography at least twice daily for the presence of fetal heart abnormalities and/or uterine contractions.

Following amniocentesis, each woman was followed prospectively to delivery. The decision for amniocentesis or delivery was made by the primary physician who was blinded to all research test results. Induction of labor or a surgical delivery was performed for such clinical indications as AF laboratory results traditionally considered to indicate intra-amniotic inflammation/ infection (17), fetal lung maturity, prolapsed umbilical cord and/or GA $\geq 34$ wk (18).

AF was cultured for aerobic and anaerobic bacteria, Ureaplasma and Mycoplasma species. The clinical laboratory performed the glucose and lactate dehydrogenase (LDH) measurements, the Gram's stain, the white (WBC) and red blood cell (RBC) counts. An AF glucose cut-off of $\leq 15$ $\mathrm{mg} / \mathrm{dL}$ (19) and LDH levels $\geq 419 \mathrm{U} / \mathrm{L}$ were considered clinically suggestive of intra-amniotic inflammation/infection (20). These results were available to the primary care providers for clinical management.

$A F$ proteomic profiling and generation of the MR score. Following $\mathrm{AF}$ retrieval, the MR score was immediately generated. The methodology for generation of the MR score has been previously described $(14,21)$. Briefly, 5 $\mu \mathrm{L}$ of $\mathrm{AF}$ was placed on spots of duplicate $\mathrm{H} 4$ arrays (8-spot $\mathrm{H} 4$ array,
Ciphergen Biosystems, Fremont, California). After $1 \mathrm{~h}$ of incubation the arrays were read in the ProteinChip Reader (Model PBS IIC) (Ciphergen Biosystems) using the ProteinChip Software 3.1.1. Peaks composing the MR score were identified by their conspicuous aspect at or in proximity of their known respective masses: 3,377.0 and 3,448.1 $\mathrm{Da}$ (corresponding to neutrophil defensins 2 and 1, respectively) and at 10,443.8 and 10,834.5 Da (corresponding to calgranulins $\mathrm{C}$ and A respectively) (14). The MR score provides qualitative information regarding the presence or absence of intraamniotic inflammation (14). The MR score ranges from 0 to 4 , depending upon the presence or absence of each of the four protein biomarkers (14). A categorical value of 1 is assigned if a biomarker peak is present and 0 if absent. For the purpose of data analysis, we stratified the study population based on the severity of inflammation (MR 0: no inflammation; MR 1-2: minimal inflammation; MR 3-4: severe inflammation). One investigator (IAB) performed all the protein chip assays and scored all the samples blindly, being unaware of either clinical presentation or outcome.

Histologic evaluation of umbilical cords and diagnosis of funisitis. At Yale-New Haven Hospital, placental pathologic study is a routine part of the evaluation of a pregnancy complicated by PTL or PPROM. In all 123 cases, hematoxylin- and eosin-stained sections of the umbilical cord were read by a perinatal pathologist unaware of the results of the proteomic profiling. Each section was examined systematically for the presence or absence of inflammation and funisitis was diagnosed when neutrophils infiltrated the umbilical vessels walls or Wharton's jelly. Funisitis results were expressed using the histologic grading system devised by Salafia et al. (22) as: Grade 0, no neutrophils observed; Grade 1, neutrophils within the inner third of the umbilical vein wall (umbilical phlebitis); Grade 2, neutrophils within the inner third of at least two umbilical vessel walls; Grade 3, neutrophils in the perivascular Wharton's jelly; or Grade 4, panvasculitis and funisitis extending deep into the Wharton's jelly.

Evaluation of early-onset neonatal sepsis. Neonatal hematological indices and sepsis categorization were assessed as previously described $(23,24)$ from blood specimens and cultures obtained after delivery by an investigator (VB) unaware of the results of the proteomic profiling or histologic evaluation for funisitis. Of all pregnancies included in the study, 97 neonates were admitted to the Newborn Special Care Unit (NBSCU) (Fig. 1). All neonates underwent clinical and laboratory evaluations for sepsis. Early-onset neonatal sepsis was defined as the presence of confirmed or suspected sepsis at $\leq 3 \mathrm{~d}$ after birth (23). Confirmed sepsis was established when either the blood and/or cerebrospinal fluid cultures were positive. Suspected sepsis was diagnosed in the presence of clinical suspicion of sepsis (signs/symptoms of which included lethargy, apnea, respiratory distress, hypoperfusion and shock) with support from laboratory results. Laboratory criteria were based on modification of the criteria of Rodwell et al. $(23,25)$ when $\geq 2$ of the following were observed: absolute neutrophil count (ANC) $<7,500 / \mathrm{mL}$ or $>14,500 / \mathrm{mL}$, absolute band count $(\mathrm{ABC})>1,500 / \mathrm{mL}$, immature/total neutrophil ratio (I:T) ratio $>16 \%$, platelet count $<150,000$ cells $/ \mathrm{mm}^{3}$ or abnormal spinal tap (23). Early-onset neonatal sepsis was dichotomized into present (when sepsis was either

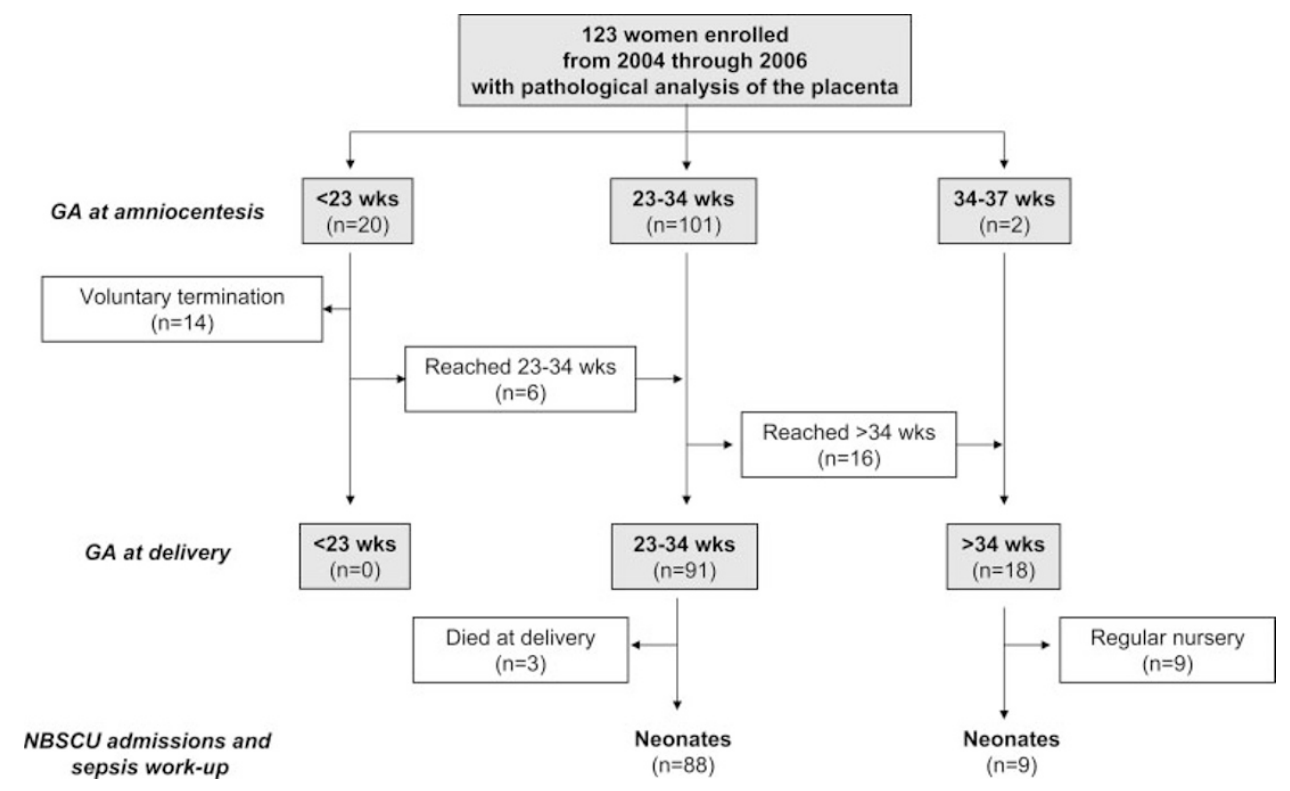

Figure 1. Selection, enrollment and follow-up of the mothers and infants in the study population (wks, weeks; GA, gestational age; NBSCU, NewBorn Special Care Unit). 
confirmed or suspected) or absent and coefficients of associations with other binary or ranked variables calculated. All neonates with confirmed or suspected sepsis received antibiotic therapy.

Statistical analysis. Statistical analyses were performed with Sigma Chemical Co. Stat, version 2.03 (SPSS Inc., Chicago, IL) and MedCalc (Broekstraat, Belgium) statistical softwares. Data were compared with MannWhitney test, one-way ANOVA followed by Dunnet's tests (parametric) or Kruskal-Wallis on ranks followed by Dunn's tests (nonparametric), to adjust for multiple comparisons as appropriate. Pearson or Spearman correlations were used to measure co-linearity between the selected independent variables as well as other relevant relationships between dependent and independent variables. Comparisons between proportions were done with $\chi^{2}$ tests. Receiver operating characteristics (ROC) curve analysis was performed using MedCalc (Broekstraat, Belgium) statistical software. A $p$-value of $<0.05$ was considered significant. Odds ratios (OR) and $p$-values were adjusted with the use of multiple stepwise logistic or linear regression analysis as appropriate. Variables were entered in the model if $p<0.05$ and removed if $p>0.1$.

\section{RESULTS}

Characteristics of women at the time of AF sampling. The clinical characteristics of the women at amniocentesis are presented in Table 1. We determined that clinical chorioamnionits and PPROM occurred more frequently in relationship to severe inflammation (MR 3-4) of the AF. Likewise, women with severe inflammation were of lower GA at recruit- ment, had shorter amniocentesis to delivery interval and delivered babies with lower birth weights compared with the no (MR 0) or mild (MR1-2) inflammation groups. Fourteen patients $(\mathrm{GA}<23 \mathrm{wk})$ elected to terminate their pregnancy when the results of the AF were highly suggestive of infection, or after counseling in regard to the risks of prematurity. Seventyseven patients delivered within $72 \mathrm{~h}$ from amniocentesis.

Women with MR scores 3-4 had lower AF glucose concentration, increased WBC count, higher LDH levels, and a higher prevalence of a positive Gram's stain or positive microbial culture result, compared with women with MR scores 0 or 1-2 (Table 2). The prevalence of a positive AF culture was $34.1 \%$ (42/123). The most common isolates were Ureaplasma urealyticum (16/42), E. coli (7/42), Bacteroides (5/42) and Prevotella species (4/42). Eleven women with positive AF cultures (26.2\%) grew multiple microbes.

Relationships between AF inflammation and histologic funisitis. The prevalence of funisitis (any grade) in our study population was $43 \%$ (53/123). We confirmed that women with funisitis had lower AF glucose concentrations, higher $\mathrm{AF}$ WBC count and total LDH activity, higher incidence of a

Table 1. Characteristics of women who had a clinically indicated amniocentesis to rule-out inflammation/infection and characteristics of their infants $(n=123)$

\begin{tabular}{|c|c|c|c|c|}
\hline Variable & $\begin{array}{c}\text { MR } 0 \\
\mathrm{n}=29\end{array}$ & $\begin{array}{c}\text { MR } 1-2 \\
n=46\end{array}$ & $\begin{array}{c}\text { MR 3-4 } \\
\mathrm{n}=48\end{array}$ & $p$ value \\
\hline \multicolumn{5}{|l|}{ Clinical characteristics at amniocentesis } \\
\hline Gestational age at recruitment: $(w k s)$, mean $\pm S D$ & $29.1 \pm 0.8$ & $27.4 \pm 5.2$ & $26.9 \pm 3.5$ & $0.101 \|$ \\
\hline Age: $(y r)$, mean $\pm S D$ & $27.3 \pm 5.7$ & $28.1 \pm 6.8$ & $28.7 \pm 7.1$ & $0.677 \|$ \\
\hline Gravidity: median [range] & $2[1-9]$ & $2[1-7]$ & $3[0-8]$ & $0.964 \dagger$ \\
\hline Parity: median [range] & $1[0-5]$ & $0[0-4]$ & $1[0-6]$ & $0.267 \dagger$ \\
\hline History of PTD: $\mathrm{n},(\%)$ & $11(37.9)$ & $10(21.7)$ & $12(25.1)$ & $0.285 \S$ \\
\hline PPROM: n, (\%) & $11(37.9)$ & $14(30.4)$ & $29(60.4)$ & $0.010 \S$ \\
\hline Advanced cervical dilatation $(\geq 3 \mathrm{~cm}: \mathrm{n},(\%)$ & $7(24.1)$ & $17(36.9)$ & $17(35.4)$ & $0.480 \S$ \\
\hline Cervical dilatation: $(\mathrm{cm})$, median [range] & $1[0-5]$ & $1.5[0-10]$ & $1.25[0-8]$ & $0.411 \dagger$ \\
\hline Uterine contractions: $\mathrm{n},(\%)$ & $11(37.9)$ & $21(45.6)$ & $23(47.9)$ & $0.685 \S$ \\
\hline Clinical chorioamnionitis: $\mathrm{n},(\%)$ & $1(3.6)$ & $4(8.7)$ & $11(22.9)$ & $0.026 \S$ \\
\hline \multicolumn{5}{|l|}{ Outcome and delivery measures } \\
\hline Amniocentesis-delivery interval: $(d)$, median [range] & $6.7[0.1-62.2]$ & $1.9[0.0-98.3]$ & $0.4[0.0-12.2]$ & $<0.001 \dagger$ \\
\hline Delivery within 72 hours: $\mathrm{n},(\%)$ & $13(44.8)$ & $25(54.3)$ & $39(81.3)$ & $0.002 \S$ \\
\hline Cesarean delivery: $\mathrm{n},(\%)$ & $9(31.1)$ & $17(36.9)$ & $19(39.5)$ & $0.751 \S$ \\
\hline Gestational age at delivery: (wks), median [range] & $33.1[20.4-38.1 .1]$ & $30.6[17.0-40.2]$ & $27.4[21.0-32.6]$ & $<0.001 \dagger$ \\
\hline Birthweight: $(g)$, median $\pm S D$ & $1,826 \pm 5.7$ & $1,599 \pm 847.1$ & $1,144 \pm 508.3$ & $<0.001 \|$ \\
\hline Apgar 1 min: median [range] & $7[0-9]$ & $7[0-9]$ & $6[0-9]$ & $0.233 \dagger$ \\
\hline Apgar 5 min: median [range] & $9[0-9]$ & $9[0-9]$ & $8[0-9]$ & $0.107+$ \\
\hline
\end{tabular}

$\|$ One-Way ANOVA; † Kruskal-Wallis ANOVA; § Chi square test.

$\mathrm{cm}=$ centimetres; wks $=$ weeks; $\mathrm{yr}=$ years; $\mathrm{d}=$ days; $\mathrm{g}=$ grams; PTD $=$ preterm delivery; PPROM $=$ preterm premature rupture of membranes.

Table 2. Amniotic fluid characteristics

\begin{tabular}{|c|c|c|c|c|}
\hline Variable & $\begin{array}{c}\text { MR } 0 \\
\mathrm{n}=29\end{array}$ & $\begin{array}{c}\text { MR } 1-2 \\
n=46\end{array}$ & $\begin{array}{c}\text { MR 3-4 } \\
\mathrm{n}=48\end{array}$ & $p$ value \\
\hline \multicolumn{5}{|l|}{ Clinical laboratory tests } \\
\hline Glucose: $(m g / d L)$, median [range] & 34 [11-98] & $26[6-53]$ & $6[2-56]$ & $<0.001 \dagger$ \\
\hline WBC: $\left(\right.$ cells $\left./ \mathrm{mm}^{3}\right)$, median [range] & $3[0-200]$ & $5[0-767]$ & $577[2-37,000]$ & $<0.001 \dagger$ \\
\hline LDH: $(U / L)$ median [range] & 118 [73-265] & $176[56-2,160]$ & $622[126-4,560]$ & $<0.001 \dagger$ \\
\hline RBC: $\left(\right.$ cells $\left./ \mathrm{mm}^{3}\right)$, median [range] & $75[0-78,500]$ & $38[0-223,000]$ & $245[0-672,000]$ & $0.055 \dagger$ \\
\hline Positive Gram stain: n, (\%) & $2(6.9)$ & $4(8.7)$ & $20(40.8)$ & $<0.001 \S$ \\
\hline Positive amniotic fluid cultures: $\mathrm{n},(\%)$ & $3(10.3)$ & $5(10.8)$ & $34(70.8)$ & $<0.001 \S$ \\
\hline
\end{tabular}

$\dagger$ Kruskal-Wallis ANOVA; § Chi-square test.

$\mathrm{mg}=$ milligrams; $\mathrm{dL}=$ deciliters; $\mathrm{mL}=$ milliliters; $\mathrm{mm}=$ millimeters $\mathrm{L}=$ liters $\mathrm{U}=$ units; $\mathrm{WBC}=$ white blood cell count $\mathrm{RBC}=$ red blood cell count; $\mathrm{LDH}=$ lactate dehydrogenase activity. 
Table 3. Amniotic fluid characteristics of women with or without histological funistis $(n=123)$

\begin{tabular}{|c|c|c|c|}
\hline Variable & $\begin{array}{c}\text { Funistis } \\
\mathrm{n}=53\end{array}$ & $\begin{array}{c}\text { No Funistis } \\
\mathrm{n}=70\end{array}$ & $p$ value \\
\hline \multicolumn{4}{|l|}{ Clinical laboratory tests } \\
\hline Glucose: $(m g / d L)$, median [range] & $10[2-52]$ & $27[2-98]$ & $<0.001$ 末 \\
\hline LDH: (U/L), median [range] & $414.5[104-4,560]$ & $174[56-2,160]$ & $<0.001$ \\
\hline RBC: $\left(\right.$ cells $\left./ \mathrm{mm}^{3}\right)$, median [range] & $96[0-223,000]$ & $90[0-672,000]$ & 0.683 车 \\
\hline Positive Gram stain: $\mathrm{n}(\%)$ & $22(41)$ & $4(5.8)$ & $<0.001 \S$ \\
\hline MR score $0: \mathrm{n},(\%)$ & $5(9.4)$ & $24(34.3)$ & $<0.001 \S$ \\
\hline MR score $1-2: \mathrm{n},(\%)$ & $12(22.6)$ & $34(48.6)$ & $0.009 \S$ \\
\hline MR score 3-4: n, (\%) & $36(67.9)$ & $12(17.1)$ & $<0.001 \S$ \\
\hline
\end{tabular}

$\ddagger$ Mann-Whitney test; § Chi-square test.

$\mathrm{mg}=$ milligrams; $\mathrm{dL}=$ deciliters; $\mathrm{mL}=$ milliliters; $\mathrm{mm}=$ millimeters $\mathrm{L}=$ liters; $\mathrm{U}=$ units; $\mathrm{WBC}=$ white blood cell count; $\mathrm{RBC}=$ red blood cell count; $\mathrm{LDH}=$ lactate dehydrogenase activity.

positive Gram's stain and positive AF culture results (Table 3). Women with abnormal MR scores (MR scores 3-4) were more likely to have histologic funisitis (Fig. 2). There was a significant correlation between the severity of AF inflammation as estimated by the MR score and the presence $(r=0.50$, $p<0.001)$ or grade $(r=0.52, p<0.001)$ of histologic funisitis. Our results thus indicate that patients with an MR score 3-4 are more likely to have marked neutrophil infiltration of the Wharton's jelly and umbilical cord vasculitis. Multivariate logistic regression analysis revealed that a diagnosis of histologic funisitis occurred independent of the amniocentesis-to-delivery interval or status of the membranes (intact versus ruptured) and was best predicted by the combination of an MR 3-4 (OR: 9.3 [95\% CI: 3.9-22.3], $p<0.001$ ) and an earlier GA at delivery (OR: 0.9 [95\% CI: 0.8-1.0], $p=$ $0.04)$. When AF WBC count was added into the model, only the MR 3-4 remained significantly associated with funisitis (OR: 11.3 [95\% CI: 4.7-27.2], $p<0.001$ ) suggesting that $\mathrm{AF}$ WBC had less value in predicting funisitis compared with the MR score. This is shown in Table 4. The relationship between the grade of funisitis and MR score is presented in Figure 3.

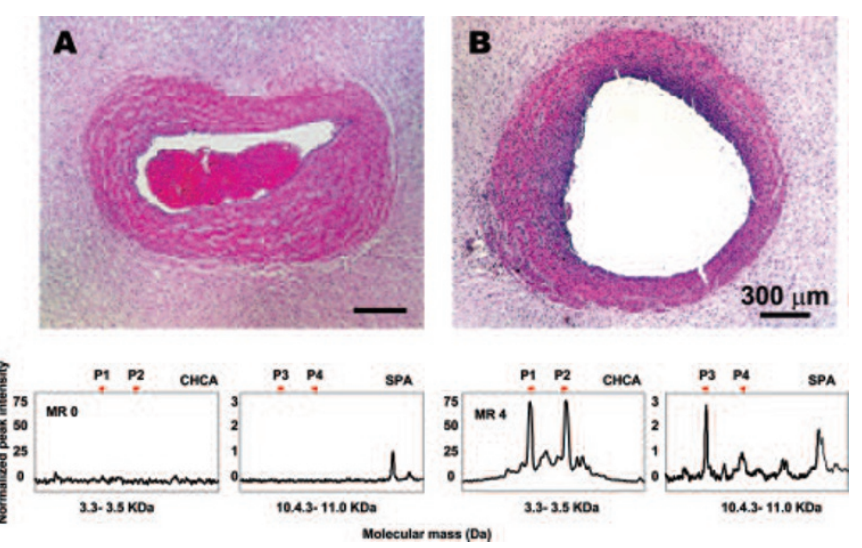

Figure 2. Representative SELDI-TOF mass spectrometry profiles of the amniotic fluid with corresponding light microscopy of hematoxylin eosin (H\&E) stain sections of the umbilical vein in a woman with MR $0(A$, no inflammation, funisitis grade 0$)$ and MR 3-4 ( $B$, severe inflammation, funisitis grade 4).
There was a significant and direct correlation between the grade of funisitis and the MR score $(r=0.537$ [95\% CI: 0.400-0.654], $p<0.001$ ).

To exclude the possibility of a change in the inflammatory status of the AF due to a prolonged amniocentesis-to-delivery interval affecting the presence of histologic funisitis, we also applied our analysis to cases delivered within $72 \mathrm{~h}$ from the time of the amniocentesis $(n=77)(26)$. For this subset analysis, an MR score 3-4, but not the AF WBC count, remained in our model as the single most significant predictor of acute funisitis (OR: 11.3 [95\% CI: 3.8-33.3], $p<0.001$ ). The direct and significant correlation of the MR score with the grades of funisitis was also maintained for this analysis $(r=$ 0.546 [95\% CI: 0.366-0.686], $p<0.001$ ).

Next, we restricted the analysis to the 97 women whose neonates were admitted to the NBSCU. MR score of 3-4 remained the sole significant predictor of histologic funisitis (OR: 12.5 [95\% CI: 4.7-33.1], $p<0.001$ ). Of all the clinical laboratory tests traditionally used to diagnose intra-amniotic inflammation/infection, the combination of MR 3-4 and a positive Gram's stain had the strongest and nonredundant association with funisitis at delivery $(n=123$; MR score 3-4 OR: 7.8 [95\% CI: 3.1-19.2], $p<0.001$ and positive Gram's stain OR: 6.2 [95\% CI: $1.8-21.6$ ], $p=0.004$ ).

Early-onset neonatal sepsis and AF analysis. The clinical characteristics of the neonatal study population with hemato-

Table 4. Stepwise logistic regression analysis in predicting funisitis

\begin{tabular}{lcclll}
\hline \multicolumn{2}{c}{ Model 1 } & & & \multicolumn{2}{c}{ Model 2 } \\
\cline { 1 - 2 } \cline { 5 - 5 } Variables & $p$ value & & \multicolumn{1}{c}{ Variables } & $p$ value \\
\hline MR 3-4 & $<0.0001$ & & MR 3-4 & $<0.0001$ \\
GA at delivery & 0.0443 & & AF WBC count & $>0.1$ \\
GA at amniocentesis & $>0.1$ & & GA at delivery & $>0.1$ \\
$\begin{array}{c}\text { Amniocentesis to } \\
\text { delivery interval }\end{array}$ & $>0.1$ & & GA at amniocentesis & $>0.1$ \\
PPROM & $>0.1$ & & Amniocentesis to & $>0.1$ \\
& & & delivery interval & $>0.1$ \\
\end{tabular}

$\mathrm{GA}=$ gestational age PPROM $=$ preterm premature rupture of membranes; $\mathrm{AF}=$ amniotic fluid; $\mathrm{WBC}=$ white blood cell count. 
logical indices are presented in Table 5. A total of 22 neonates had confirmed/suspected sepsis. Of these, 3 neonates had a positive blood culture result. One fetus was delivered from a mother with an MR of 4 and the other 2 from pregnancies with MR scores of 2. Overall, neonates delivered from mothers with AF MR scores of 3-4 were born earlier and had lower birth weights compared with the babies delivered by women with MR 0 or 1-2. Furthermore, the neonates of women with MR score 3-4 were more often lymphocytopenic $(p=0.007)$, had significantly higher percentage of bandemia $(p<0.001)$, absolute band count $(p<0.001)$, I:T ratio $(p<0.001)$, and increased incidence of suspected and/or confirmed sepsis (odds ratio: 4.0 [95\% CI: 1.5-11.1], $p=0.007$ ) compared with neonates from mothers with MR 0 or 1-2, even after adjusting for GA. Neonatal suspected and/or confirmed sepsis associated significantly with the MR score $(r=0.31, p=$ 0.002). In bivariate logistic regression analysis, an MR 3-4, but not the AF WBC count, was significantly associated with neonatal sepsis (MR 3-4 OR: 4.2 [95\% CI: 1.5-11.7]).

Relationships between funisitis, early-onset neonatal sepsis and proteomic biomarkers. The prevalence of funisitis was $41.2 \%$ (40/97). By using ROC analyses, we found that only an

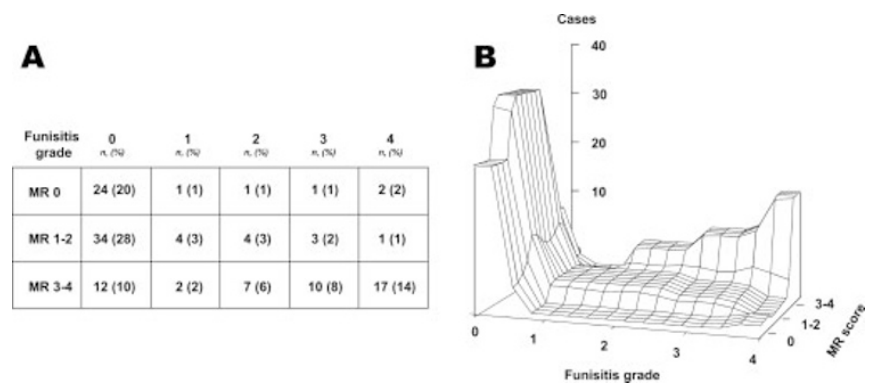

Figure 3. Distribution of grades of funisitis $(0-4)$ as a function of the MR score (MR 0: no inflammation; MR 1-2: minimal inflammation; MR 3-4: severe inflammation $(A)$ and 3-D-mesh interpolation of the distribution $(B)$. advanced grade of funisitis (grades 2-4) had clinical relevance from the perspective of hematological markers indicating early-onset sepsis (sensitivity: 73\% [95\% CI: 50-89], specificity: $75 \%$ [95\% CI: 63-84], positive predictive value (PPV): 46\% [95\% CI: 63-84]; negative predictive value (NPV): 90\% [95\% CI: 80-96]; accuracy 74\%). We first determined that out of all patients with MR score 3-4, 62.5\% (30/48) had clinically significant funisitis (i.e., relationship with early-onset neonatal sepsis). This was significantly higher compared with women with MR score $0(19.2 \%)(5 / 21)$ or MR score 1-2 (22.5\%) (9/31) $(p<0.001)$. Because of our previous findings suggesting the sequential appearance of biomarkers in the progress of intra-amniotic inflammation (14), we next aimed to determine the appearance of which of the 4 proteomic biomarkers had the strongest relationship with funisitis and/or development of early-onset neonatal sepsis. Our analysis revealed that of all the component biomarkers of the MR score, the presence of calgranulin C (Table 6A) had the highest association with the occurrence of the relevant grade of funisitis (grades 2-4) at the time of delivery (OR: 16.7 [95\% CI: 5.9-47.2], $p<0.001, n=97$ ) while the presence of calgranulin A retained the strongest association with occurrence of neonatal sepsis (OR: 4.8 [95\% CI: $1.7-$ 13.2], $p=0.002, n=97$ ) (Table 6B).

\section{DISCUSSION}

Our research was motivated by the fact that histologic chorioamnionitis and funisitis has been previously linked with neonatal morbidity in preterm infants $(27,28)$. We have previously shown that proteomic profiling of the AF can rapidly and accurately diagnose intra-amniotic inflammation, and can identify women at risk of premature delivery (14). We further demonstrated that interpretation of the MR score is a gradient progressing from the absence of disease to mild and then severe inflammation. In this study, we demonstrate that

Table 5. Characteristics and hematologic indices of neonates admitted to the Newborn Special Care Unit $(n=97)$

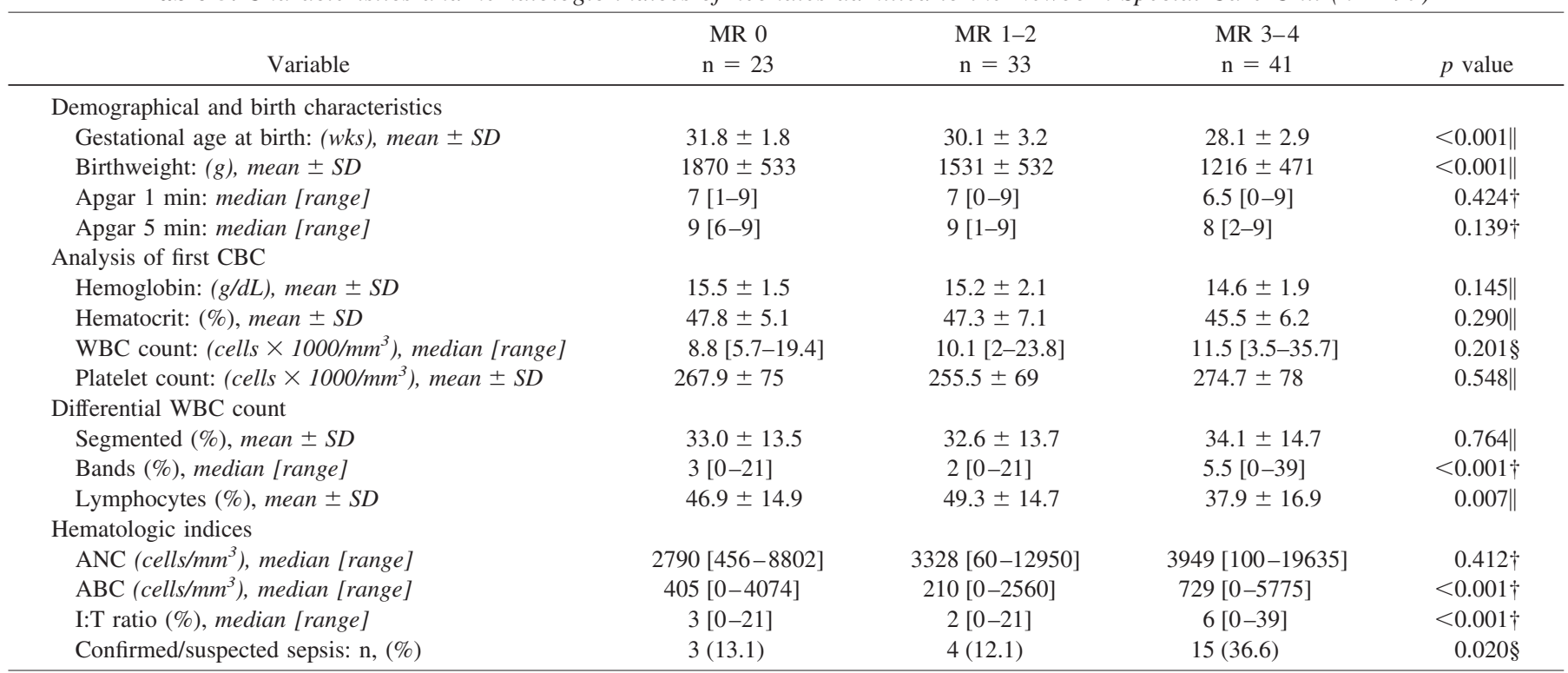

$\|$ One-Way ANOVA; † Kruskal-Wallis ANOVA; § Chi square.

wks = weeks; $\mathrm{g}=$ grams; $\mathrm{ANC}=$ absolute neutrophil count; $\mathrm{ABC}=$ absolute band count; I:T ratio $=$ proportion of immature-to-total neutrophils. 
Table 6. Performances of the four proteomic biomarkers in identifying funisitis and suspected/confirmed neonatal sepsis

\begin{tabular}{lcccc}
\hline & $\begin{array}{c}\text { Sens. } \\
{[95 \% \mathrm{CI}]}\end{array}$ & $\begin{array}{c}\text { Spec. } \\
{[95 \% \mathrm{CI}]}\end{array}$ & + PV & $\begin{array}{c}\text { Accuracy } \\
(\%)\end{array}$ \\
\hline A Funisitis & & & & $-\mathrm{PV}$ \\
P1 - defensin 2 & $91[76-98]$ & $34[22-47]$ & $44[32-55]$ & $87[67-97]$ \\
P2 - defensin 1 & $91[76-98]$ & $50[37-62]$ & $51[37-63]$ & $91[76-98]$ \\
P3 - Calgranulin C & $80[63-91]$ & $81[68-89]$ & $70[53-83]$ & $88[76-94]$ \\
P4 - Calgranulin A & $46[28-63]$ & $82[70-90]$ & $59[38-77]$ & $73[60-82]$ \\
B Neonatal Sepsis & & & & 85 \\
P1 - defensin 2 & $86[65-97]$ & $28[18-39]$ & $26[16-37]$ & $88[67-97]$ \\
P2 - defensin 1 & $82[59-94]$ & $40[28-51]$ & $29[17-41]$ & $88[72-96]$ \\
P3 - Calgranulin C & $68[45-86]$ & $67[54-77]$ & $38[22-54]$ & $88[76-94]$ \\
P4 - Calgranulin A & $55[32-75]$ & $80[69-88]$ & $44[25-64]$ & $86[75-92]$ \\
\hline
\end{tabular}

women with abnormal MR scores are more likely to have histologic funisitis. We further determined that there is a direct relationship between the degree of intra-amniotic inflammatory response, as estimated by the MR score (but not WBC, when both were included in the model) and the severity of umbilical cord vasculitis. This relationship maintained for fetuses delivered within $72 \mathrm{~h}$ from amniocentesis. Finally, we showed that infiltration of the umbilical cord by inflammatory cells is most accurately predicted by the presence of calgranulin $\mathrm{C}$, while a more advanced degree of inflammation as reflected by the presence of calgranulin $\mathrm{A}$ is predictive of early-onset neonatal sepsis.

Sepsis occurs with increased frequency in the premature neonate $(12,29,30)$. Laboratory tests including IL-6, MMP-8 and WBC counts have been proposed as a means to predict a fetal inflammatory response to infection or other inflammatory triggers (bleeding). However, such tests remain poor instruments for clinical decision-making, as they either require an invasive fetal blood sampling (cordocentesis), or laborious immunoassay laboratory protocols. Regrettably, although histologic funisitis remains a reliable diagnostic marker of neonatal sepsis, its presence cannot be recognized before delivery of the fetus. The strong association between early-onset neonatal sepsis, and elevated cytokines levels in the fetal circulation warrants investigation into selection of a panel of diagnostic markers aimed to rapidly and accurately identify infants with severe inflammatory syndrome and therefore, adverse prognosis before delivery $(2,12,23)$.

Today, an emerging field, proteomics, seeks to apply the science of functional biology in the search for biomarkers and generation of protein profiles that can rapidly predict and diagnose human and fetal diseases, including PTB and fetal inflammatory syndrome (31). The use of specific biomarkers to identify neonates with increased risk for sepsis in utero, would aid in initiation of appropriate therapy and perhaps decrease the risk of resistant microorganisms by eliminating unnecessary antibiotic treatment (30). Our finding creates the premise for prospective studies, in larger cohorts of neonates, to validate our conclusions drawn about the association of higher MR scores with neonatal sepsis.

In our current study, we found that proteomic analysis of the AF-identified women with severe inflammation who were more likely to have histologic funisitis of a more advanced grade. Consistent with other prior studies, we further confirmed that the presence or absence of histologic funisitis is unrelated to the amniocentesis-to-delivery interval (32). Therefore, at the time of the amniocentesis, and thus before delivery of the fetus, our data would suggest that the clinician may be either reassured in the absence of inflammation (MR 0 ), or initiate aggressive therapy immediately following delivery in the context of severe inflammation (MR 3-4) on the assumption of a high probability of funisitis and fetal sepsis when inflammatory biomarkers are identified in the AF. We found out that a significantly higher number of patients with MR score 3-4 had clinically significant funisitis (grades 2-4) and suspected or confirmed, early-onset neonatal sepsis, when compared with women with MR scores 0 or 1-2. Early recognition, diagnosis and treatment of neonatal sepsis is an important aspect of the current clinical practice and hence, prospective randomized studies aimed to determine the appropriate treatment of the newborn based on the MR score is highly desirable (33).

We further found that only an advanced grade of funisitis (grades 2-4) is of clinical relevance for the neonate. Indeed, neutrophilic infiltration of the umbilical artery, and thus an advanced stage of funisitis, has been shown to be associated with a higher fetal blood concentration of inflammatory cytokines and adverse neonatal outcome (34). So far, while such findings provide answers to important physiologic questions pertaining to fetal inflammatory response, our discovery that funisitis is accurately predicted by the presence of the AF biomarker calgranulin $\mathrm{C}$ offers practical directions relevant for a rapid antenatal diagnosis. Presence of calgranulin A was predictive of early neonatal sepsis. Because the monomeric calgranulin $\mathrm{A}$ is not reliably estimated by immunoassays (ELISA) as it binds to calgranulin B to form the calprotectin complex, mass spectrometry remains the only way to discriminate between biomarker isoforms at this time (35). The observation that calgranulin $\mathrm{C}$ is strongly associated with funisitis while calgranulin $\mathrm{A}$ is linked with neonatal sepsis (a more advanced stage of disease) suggests that there is a specific temporal sequence in the appearance of these biomarkers in women with intra-amniotic infection/inflammation.

Our study is novel and puts forward the idea that recognition of proteomic biomarkers characteristic of intra-amniotic inflammation can predict not only the presence or absence of clinical significant funisitis antenatally, but can provide the clinician with more complete, integrative information pertaining to the risk of preterm delivery, the state of functional 
activation of the infiltrative neutrophil, and early-onset neonatal sepsis.

Acknowledgments. We are indebted to the nurses, residents, and fellows from the Department of Obstetrics, Gynecology and Reproductive Sciences, and Department of Pediatrics, Division of Perinatal Medicine at Yale New Haven Hospital and to all patients who participated in the study.

\section{REFERENCES}

1. Hoyert DL, Mathews TJ, Menacker F, Strobino DM, Guyer B 2006 Annual summary of vital statistics: 2004. Pediatrics 117:168-183

2. Green NS, Damus K, Simpson JL, Iams J, Reece EA, Hobel CJ, Merkatz IR, Greene MF, Schwarz RH March Of Dimes Scientific Advisory Committee On Prematurity. 2005 Research agenda for preterm birth: recommendations from the March of Dimes. Am J Obstet Gynecol 193:626-635.

3. Lau J, Magee F, Qiu Z, Hoube J, Von Dadelszen P, Lee SK 2005 Chorioamnionitis with a fetal inflammatory response is associated with higher neonatal mortality, morbidity, and resource use than chorioamnionitis displaying a maternal inflammatory response only. Am J Obstet Gynecol 193:708-713

4. Buhimschi IA, Buhimschi CS, Weiner CP 2003 Protective effect of N-acetylcysteine against fetal death and preterm labor induced by maternal inflammation. Am J Obstet Gynecol 188:203-208

5. Eschenbach DA 1997 Amniotic fluid infection and cerebral palsy. Focus on the fetus. JAMA 278:247-248

6. Yoon BH, Park CW, Chaiworapongsa T 2003 Intrauterine infection and the development of cerebral palsy. BJOG 110:124-127

7. Salafia CM, Sherer DM, Spong CY, Lencki S, Eglinton GS, Parkash V, Marley E, Lage JM 1997 Fetal but not maternal serum cytokine levels correlate with histologic acute placental inflammation. Am J Perinatol 14:419-422

8. Rogers BB, Alexander JM, Head J, McIntire D, Leveno KJ 2002 Umbilical vein interleukin-6 levels correlate with the severity of placental inflammation and gestational age. Hum Pathol 33:335-340

9. Yoon BH, Romero R, Park JS, Kim M, Oh SY, Kim CJ, Jun JK 2000 The relationship among inflammatory lesions of the umbilical cord (funisitis), umbilical cord plasma interleukin 6 concentration, amniotic fluid infection, and neonatal sepsis. Am J Obstet Gynecol 183:1124-1129

10. Lee SD, Kim MR, Hwang PG, Shim SS, Yoon BH, Kim CJ 2004 Chorionic plate vessels as an origin of amniotic fluid neutrophils. Pathol Int 54:516-522

11. Kim CJ, Yoon BH, Kim M, Park JO, Cho SY, Chi JG 2001 Histo-topographic distribution of acute inflammation of the human umbilical cord. Pathol Int 51:861865

12. Ng PC, Lam HS 2006 Diagnostic markers for neonatal sepsis. Curr Opin Pediatr 18:125-131

13. Martius JA, Roos T, Gora B, Oehler MK, Schrod L, Papadopoulos T, Gross U 1999 Risk factors associated with early-onset sepsis in premature infants. Eur J Obstet Gynecol Reprod Biol 85:151-158

14. Buhimschi IA, Christner R, Buhimschi CS 2005 Proteomic biomarker analysis of amniotic fluid for identification of intra-amniotic inflammation. BJOG 112:173-181

15. Weiner CP, Lee KY, Buhimschi CS, Christner R, Buhimschi IA 2005 Proteomic biomarkers that predict the clinical success of rescue cerclage. Am J Obstet Gynecol 192:710-718

16. Lewis DF, Bergstedt S, Edwards MS, Burlison S, Gallaspy JW, Brooks GG, Adair CD 1997 Successful magnesium sulfate tocolysis: is "weaning" the drug necessary? Am J Obstet Gynecol 177:742-745
17. Romero R, Yoon BH, Mazor M, Gomez R, Gonzalez R, Diamond MP, Baumann P, Araneda H, Kenney JS, Cotton DB, Sehgal P 1993 A comparative study of the diagnosis performance of amniotic fluid performance of amniotic fluid glucose, white blood cell count, interleukin-6, and Gram stain in the detection of microbial invasion in patients with preterm premature rupture of the membranes. Am J Obstet Gynecol 169:839-851

18. Naef RW Allbert JR, Ross EL, Weber BM, Martin RW, Morrison JC 1998 Premature rupture of membranes at 34 to 37 weeks' gestation: aggressive versus conservative management. Am J Obstet Gynecol 178:126-130

19. Edwards RK, Clark P, Locksmith Gregory J, Duff P 2001 Performance characteristics of putative tests for subclinical chorioamnionitis. Infect Dis Obstet Gynecol 9:209-214

20. Garry D, Figueroa R, Aguero-Rosenfeld M, Martinez E, Visintainer P, Tejani N 1996 A comparison of rapid amniotic fluid markers in the prediction of microbial invasion of the uterine cavity and preterm delivery. Am J Obstet Gynecol 175:13361341

21. Buhimschi IA, Buhimschi CS, Christner R, Weiner CP 2005 Proteomics technology for the accurate diagnosis of inflammation in twin pregnancies. BJOG 112:250-255

22. Salafia CM, Weigl C, Silberman L 1989 The prevalence and distribution of acute placental inflammation in uncomplicated term pregnancies. Obstet Gynecol 73:383389

23. Smulian JC, Bhandari V, Campbell WA, Rodis JF, Vintzileos AM 1997 Value of umbilical artery and vein levels of interleukin- 6 and soluble intracellular adhesion molecule-1 as predictors of neonatal hematologic indices and suspected early sepsis. J Matern Fetal Med 6:254-259

24. Rodwell RL, Leslie AL, Tudehope DI 1988 Early diagnosis of neonatal sepsis using a hematologic scoring system. J Pediatr 112:761-767

25. Rodwell RL, Taylor KM, Tudehope DI, Gray PH 1993 Hematologic scoring system in early diagnosis of sepsis in neutropenic newborns. Pediatr Infect Dis J 12:372-376

26. Park JS, Romero R, Yoon BH, Moon JB, Oh SY, Han SY, Ko EM 2001 The relationship between amniotic fluid matrix metalloproteinase-8 and funisitis. Am J Obstet Gynecol 185:1156-1161

27. Wu YW, Colford JM 2000 Chorioamnionitis as a risk factor for cerebral palsy: A meta-analysis. JAMA 284:1417-1424.

28. Leviton A, Paneth N, Reuss ML, Susser M, Allred EN, Dammann O, Kuban K, Van Marter LJ, Pagano M, Hegyi T, Hiatt M, Sanocka U, Shahrivar F, Abiri M, Disalvo D, Doubilet P, Kairam R, Kazam E, Kirpekar M, Rosenfeld D, Schonfeld S, Share J, Collins M, Genest D, Shen-Schwarz S 1999 Maternal infection, fetal inflammatory response, and brain damage in very low birth weight infants. Developmental Epidemiology Network Investigators. Pediatr Res 46:566-575

29. Stoll BJ, Hansen NI, Adams-Chapman I, Fanaroff AA, Hintz SR, Vohr B, Higgins RD National Institute of Child Health and Human Development Neonatal Research Network 2004 Neurodevelopment and growth impairment among extremely low birth weight infants is associated with poor neonatal infection. JAMA 292:23572365.

30. Gonzalez BE, Mercado CK, Johnson L, Brodsky NL, Bhandari V 2003 Early markers of late-onset sepsis in premature neonates: clinical, hematological and cytokine profile. J Perinat Med 31:60-68

31. Buhimschi CS, Weiner CP, Buhimschi IA 2006 Clinical proteomics: A novel diagnostic tool for the new biology of Preterm Labor, Part I: Proteomics tool. Obstet Gynecol Surv 61:481-486

32. Salafia CM, Ghidini A, Sherer DM, Pezzullo JC 1998 Abnormalities of the fetal heart rate in preterm deliveries are associated with acute intra-amniotic infection. J Soc Gynecol Investig 5:188-191

33. Watterberg K 2006 Anti-inflammatory therapy in the neonatal intensive care unit: present and future. Semin Fetal Neonatal Med 11:378-384

34. Kim CJ, Yoon BH, Romero R, Moon JB, Kim M, Park SS, Chi JG 2001 Umbilical arteritis and phlebitis mark different stages of the fetal inflammatory response. Am J Obstet Gynecol 185:496-500

35. Buhimschi IA, Buhimschi CS, Weiner CP, Kimura T, Hamar BD, Sfakianaki AK, Norwitz ER, Funai EF, Ratner E 2005 Proteomic but not enzyme-linked immunosorbent assay technology detects amniotic fluid monomeric calgranulins from their complexed calprotectin form. Clin Diagn Lab Immunol 12:837-844 